\title{
Understanding disparity on the Canadian prairies: A step toward improving tuburculosis outcomes
}

\author{
Victoria Cook MD ${ }^{1}$, Donald Enarson $\mathrm{MD}^{2}$, Shawna Buccholz BScN MPH${ }^{1}$
}

In the current issue of the Canadian Respiratory Journal, Long et al (1) (pages 223-230) describe the epidemiology of active tuberculosis (TB) in the prairie provinces of Canada. This retrospective analysis of cases over five years found higher rates of TB in Registered Indians compared with other Canadian-born individuals, with notification rates increasing from west to east - somewhat counter-intuitive to the historical understanding of how this disease was believed to have spread across Canada. In Alberta, rates of TB in Registered Indians on-reserve were higher than rates off-reserve and the overall proportion of TB cases contributed by Registered Indians was the lowest of the three provinces, reflecting the greater number of foreign-born cases in this province. Saskatchewan had the highest rate of TB cases in Métis, rates of TB on-reserve were higher than off-reserve and the number of pediatric cases suggests ongoing transmission in communities. In Manitoba, TB rates were most markedly highest in Registered Indians but not exclusively in those living off-reserve in Winnipeg, with many cases in that city linked to a high burden community of origin. The article also reported on a prospective analysis of the notification rates of Canadian-born culture-positive pulmonary TB cases in relation to place of residence according to latitude. There was a strong association with residence north of the 53rd parallel. In some communities, rates were on par with those found in developing countries (300 per 100,000$)$. The distribution of cases highlights the focal nature and burden of TB, with concentrations in isolated northern communities.

These findings confirm the heterogeneity of TB in the prairies with varying rates across provinces. The disparity may be explained, in part, by community environment and housing including number and size of treaty lands, characteristics of shared space, and clustering of houses with populations living on and/or off reserve. The association with latitude could reflect challenges to accessing health care. Certainly not all Aboriginal communities experience the same burden of TB disease. It is not clear why some communities have been successful in sustaining $\mathrm{TB}$ rate reduction with current $\mathrm{TB}$ programming while others have not. If TB rates in a small number of communities explain the majority of $\mathrm{TB}$ disease in Aboriginals, programming must be tailored to the community-specific needs of these highest-burden communities.

Until 2000, case rates on reserve were falling and are now considered to be 'stable'. Stability should be interpreted as stagnancy because the rates of TB in the foreign-born and Canadian-born nonAboriginal groups continue to decline. Outbreaks and a large pool of latent infection are often used to explain these persistently higher rates. However, challenges to TB control in Aboriginal populations can be split into those particular to the disease (ie, nonspecific symptoms leading to diagnostic delay, diagnostic accuracy of testing for TB infection, acceptance and completion of latent TB infection treatment, comorbid conditions and the social determinants of health) and those that may be more specific to Aboriginal communities including suboptimal access to health care, nature and extent of coordination among health care managers at various levels, cultural sensitivity of health services, health care worker staffing and retention, and geographical isolation, as well as the stigma and discrimination associated with TB that is inextricably linked to colonization, the sanatorium era and residential schools. Additional challenges to TB control in some Aboriginal communities may be the unique social and language networks, the relative mobility of community members, and the shared responsibility and funding structure for $\mathrm{TB}$ programming. It is also unclear how the broader economy impacts TB with pressures on employment, programs, housing, migration and the desire to maintain connections to homelands.

In 1992, the then Medical Services Branch of Health Canada released a 'National Tuberculosis Elimination Strategy' with a lofty elimination goal for 2010, a target that was never to be reached. In 2005, Canada adopted the goal of the WHO Global Plan to Stop TB to reducing the national rate of TB to 3.6 per 100,000 . Released in 2012, Health Canada's Strategy Against Tuberculosis for First Nations On-Reserve is designed to contribute to the national goal by reducing TB rates on-reserve (2). This strategy, albeit comprehensive and well constructed, is restricted by the absence of a united TB control plan to include First Nations persons living off-reserve, the Inuit and Métis. The heterogeneity of Aboriginal TB and the disparities noted on the prairies will be difficult to address without a collaborative approach among First Nations, provincial/territorial and federal partners. However, this research clearly shows the willingness of Aboriginal partners to support collaborative research that informs on the patterns and distribution of TB that ultimately demand a cooperative intervention.

Moving forward requires an acknowledgement and understanding that the TB profile of Aboriginal communities varies across the provinces and territories and is as diverse as the communities themselves. It also necessitates recognition of the negative aspects of the history of relationships between TB services and Aboriginal communities. A successful approach needs to be culturally sensitive, evidence-based, transparent and collaborative (3). The communities must be engaged in the development, implementation and evaluation of individualized programs to ensure shared control of outcomes. Programs may take advantage of innovations in TB control, such as social network analysis, to enhance the success of contact investigation and genotype/ genomic analysis to better understand transmission networks. However, in other areas, development of truly collaborative partnership networks among stakeholders accompanied by community-based TB education campaigns geared toward elders, youth and other at-risk community members may better facilitate screening and surveillance activities as core prevention and health promotion strategies.

\section{REFERENCES}

1. Long R, Hoeppner V, Orr P, et al. Marked disparity in the epidemiology of tuberculosis among Aboriginal peoples on the Canadian prairies: The challenges and opportunities.

Can Respir J 2013;20:223-30.

2. Health Canada's Strategy Against Tuberculosis for First Nation On-Reserve. <http://hc-sc.gc.ca/fniah-spnia/pubs/diseases-maladies/_ tuberculos/tuberculos-strateg/index-eng.php > (Accessed March 1, 2013).

3. Boffa J, King M, McMullin K, Long R. A process for the inclusion of Aboriginal People in health research: Lessons from the Determinants of TB Transmission project. Soc Sci Med 2011;72:733-8.

${ }^{1}$ TB Control, BC Centre for Disease Control, Vancouver, British Columbia; ${ }^{2}$ International Union Against Tuberculosis and Lung Disease, Paris, France

Correspondence: Dr Victoria Cook, TB Control, BC Centre for Disease Control, 655 West 12th Avenue, Vancouver, British Columbia V5Z 4R4.

Telephone 604-707-2696, fax 604-707-2690, e-mail victoria.cook@bccdc.ca 


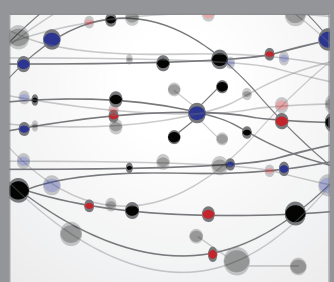

The Scientific World Journal
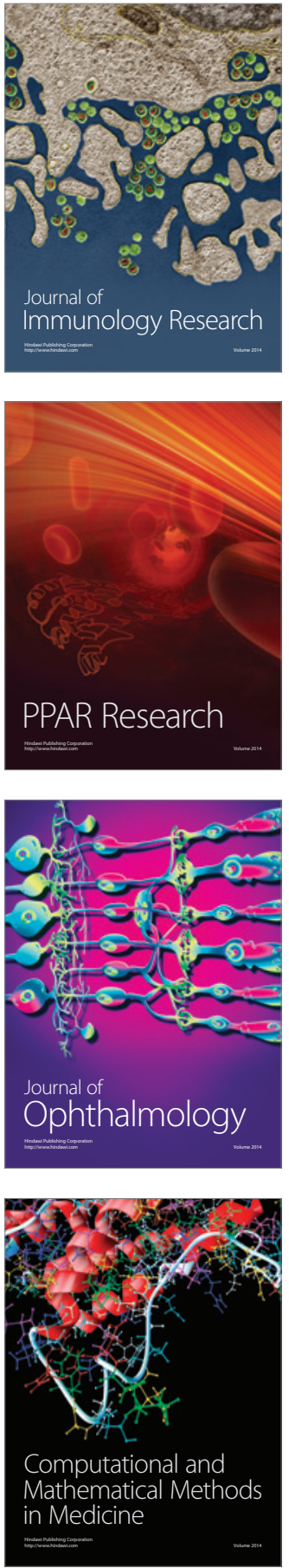

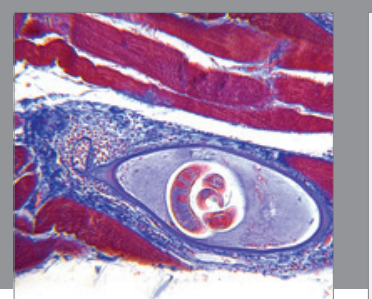

Gastroenterology Research and Practice

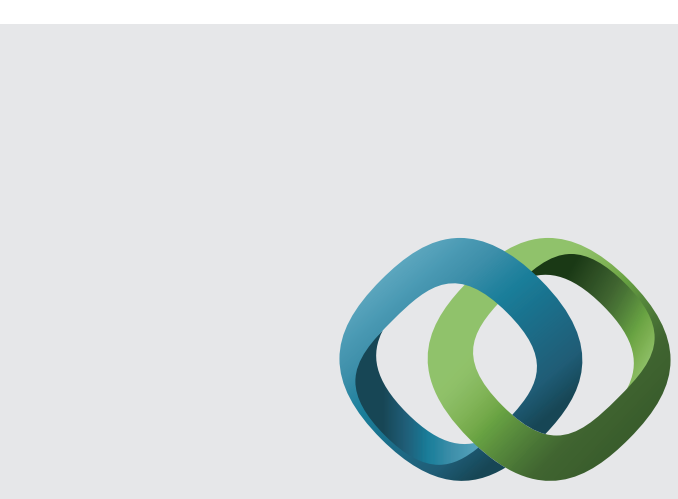

\section{Hindawi}

Submit your manuscripts at

http://www.hindawi.com
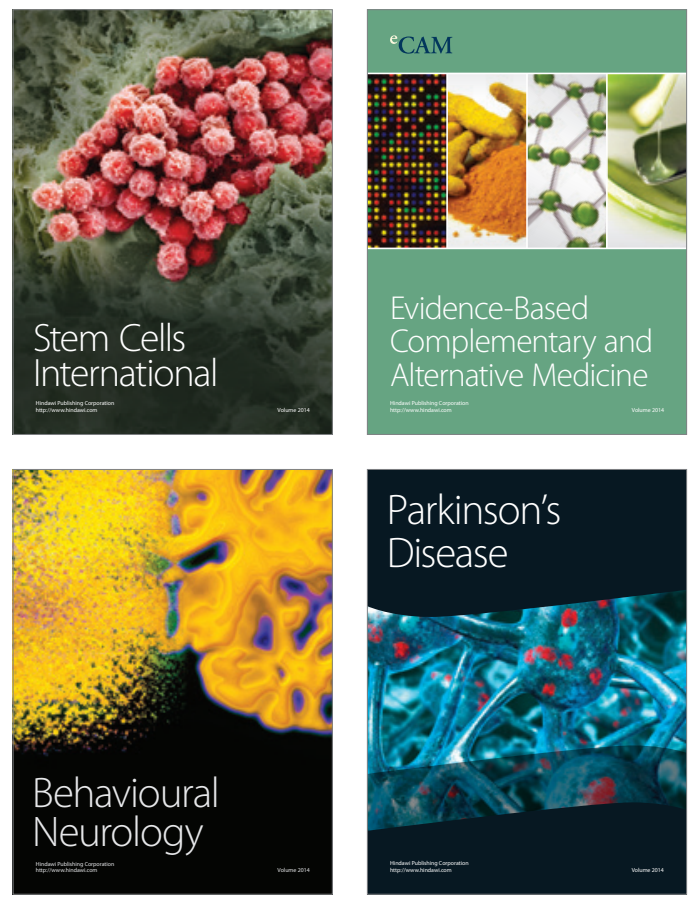
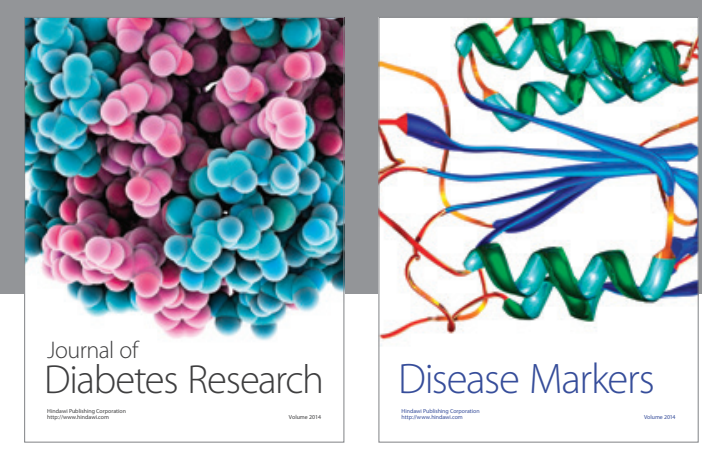

Disease Markers
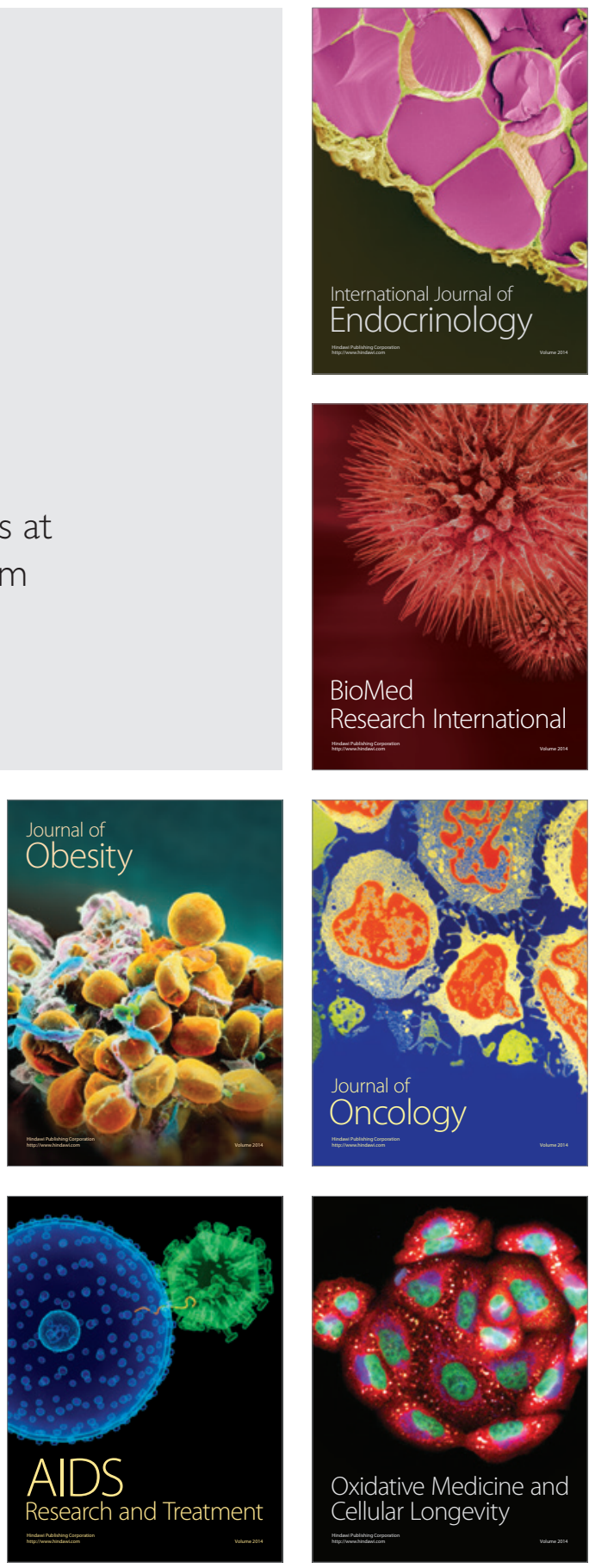